\title{
Stochastic load generated by the beam irregularities
}

\author{
Piotr Koziol ${ }^{1, *}$, Dariusz Kudla ${ }^{1}$, and Cristinel Mares ${ }^{2}$ \\ ${ }^{1}$ Faculty of Civil Engineering, Cracow University of Technology, ul. Warszawska 24, 31-155, Kraków, \\ Poland \\ ${ }^{2}$ Brunel University, Kingston Ln, London, Uxbridge UB8 3PH, United Kingdom
}

\begin{abstract}
The problem investigated in this paper comes from railway engineering. It is known that geometrical irregularities of the rail head rolling surface produce additional force when the train runs on track. This force can be quite significant and should not be neglected in the analysis, especially when one deals with high-speed railways. In this paper, an analytical method of modelling of such irregularities is presented. The detailed description of this method is associated with its practical application to the analysis of the rail track dynamic response to moving train. However, stochastic analysis of the presented model is omitted in this paper and left for further work. This should include a number of realisations along with statistical analysis of results, or description of the rail track subjected to moving train in terms of stochastic differential equations, which is the main direction of the authors future investigations.
\end{abstract}

\section{Introduction}

Modelling of dynamic loads appearing on rail tracks is quite difficult subject. Each load produced by train, contains a few terms, depending on vehicle and track conditions. The mechanical and physical properties of the systems are very often nonlinear. Changes of characteristics of the track loading are usually undetermined and vary in time. The very good example of such conditions are irregularities appearing on rail head rolling surface and wheels of train. These numerous examples lead to the necessity of consideration of more complex description of both the rail track systems and the loads generated by moving train. These descriptions should take into account nonlinear and stochastic properties.

The rail track is usually modelled by a system of beams representing rails, sleepers and other components of rail track [1-4]. The load can be modelled in various ways, as a point load or distributed one, with assumed distribution of this load, or a set of forces arranged in a way associated with the train axles configuration [1-4]. This paper deals with modelling of random part of this load, generated by irregularities appearing on the rail head rolling surface. An efficient approximation method allowing to introduce the randomized beam surface imperfections in analytically solved system of differential equations is shown and described in details.

\footnotetext{
* Corresponding author: pkoziol@pk.edu.pl
} 


\section{Rail track}

Usually when one deals with analytical approach, the rail track is modelled as a beam or a system of beams resting on elastic or viscoelastic foundation. The simplified model of track based on a beam-foundation system can be described in many ways. The simplest one uses the dynamic Euler-Bernoulli or Timoshenko beam equations and assumes that the beam rests on viscoelastic foundation. The beam can be finite or infinitely long. This assumption determines a use of appropriate techniques during solution procedure. The finite beam allows using the Fourier series and, consequently, gives more detailed information about frequencies appearing in the system response. However, in case of nonlinear properties of structure, it leads to difficulties and, actually, reflects necessity of intense calculations because of the fact that the system must be solved separately for each term of series. These difficulties become even more important in case of stochastic formulation, when some properties are described in terms of stochastic functions with constant or varying characteristics. In order to avoid these difficulties, one can consider infinitely long beam. This allows to use the Fourier transform which is much easier to apply, even if its application is needed in relation to several variables. Of course, this solution is not perfect, because information about the response frequency is limited and even impossible when analysed in the transform domain only. Therefore the next step might be sought in the application of the wavelet transforms, mainly due to their ability of features separation in time-frequency domain. For some wavelets, constructed similarly to fractals, a deeper look in strongly dynamic variations is possible, described in some papers as "digital zoom". This ability led to some important results in the field, when foundation was described by ground equation. In this case, the whole system is represented by a number of differential equations and appropriate boundary conditions. This makes its solution difficult and forces using so called semi-analytical methods (hybrid techniques based on analytical calculations combined with numerical methods and different kind of approximations).

In case of the beam resting on viscoelastic foundation (usually the Winkler one), these methods appeared very effective in finding nonlinear solutions. The Fourier transform along with the Adomian's decomposition and analytical wavelet based approximation gave new results taking into account nonlinear foundation stiffness $[4,5]$. Extension of the rail track modelling to multilayer models, where the beam represents rails but also describes sleepers considered as a rigid body, can be also solved by using the developed semi-analytical approach. The development of new computational techniques allowed to solve some simplified stochastic systems, where the randomness of foundation stiffness was assumed [6].

One can find also more complex models where the structures consisting of beams rest on multilayer foundation [7]. Each layer of this foundation may have different geometrical and mechanical properties and is usually described by separated equation of ground motion. This case, however, is difficult to solve analytically and therefore numerical methods are preferred, so far.

In this paper, the simplest one layer model of rail track is considered in order to apply the newly developed algorithm for random force generation. This force is assumed to arise from the beam irregularities.

The rail track is represented by the following dynamic partial differential equation:

$$
E I \frac{\partial^{4} y_{r}}{\partial x^{4}}+N \frac{\partial^{2} y_{r}}{\partial x^{2}}+m_{r} \frac{\partial^{2} y_{r}}{\partial t^{2}}+c_{r} \frac{\partial y_{r}}{\partial t}+k_{r} y_{r}+k_{N} y_{r}^{3}=P(x, t)
$$

in which $E I\left[\mathrm{~N} / \mathrm{m}^{2}\right]$ is a bending stiffness of rail steel, $N[\mathrm{~N}]$ is an axial force, $m_{r}[\mathrm{~kg} / \mathrm{m}]$ is a rail unit mass, $k_{r}\left[\mathrm{~N} / \mathrm{m}^{2}\right]$ is a rail foundation stiffness, $c_{r}\left[\mathrm{Ns} / \mathrm{m}^{2}\right]$ is a viscous damping of rail foundation and $k_{N}\left[\mathrm{~N} / \mathrm{m}^{4}\right]$ describes a nonlinear part of stiffness of track bed. 
This one layer model is usually considered as exact enough when one deals with linear problem and vertical vibrations of rails.

\section{The load}

In several published papers, the load generated by train is described as a set of forces concentrated or distributed with density $d(x, t)$ :

$$
\begin{gathered}
P(x, t)=P_{S}(x, t)+P_{D}(x, t)+P_{R}(x, t) \\
P_{S}(x, t)=\sum_{l=0}^{L} \frac{P_{0}}{a} d(x, t) H\left(a^{2}-\left(x-V t-s_{l}\right)^{2}\right) \\
P_{D}(x, t)=\sum_{l=0}^{L} \frac{\Delta P}{a} e^{i \omega} d(x, t) H\left(a^{2}-\left(x-V t-s_{l}\right)^{2}\right) .
\end{gathered}
$$

The term $P_{R}(x, t)$ describes factor associated with random irregularities of rail head rolling surface [8]. $H(),. 2 a, s_{l}, L, \omega$ and $V$ are the Heaviside function, the span of single load, the distance of specific axle from the front of the load (the distance between forces produced by wheels), the number of axles, the frequency of load and the speed of train, respectively.

When the rail imperfection is assumed as the regular one, e.g. described by a cosine function, the phase shift related to the wheel position on this irregularity can be easily introduced for better reflection of realistic situation. In the case of regular spacing between sleepers, this imperfection can be interpreted as rail deflection and then the amplitude of this regular cosine shape is constant $[1,4]$.

Different types of loads can be considered, e.g. rectangular, Gaussian, cosine type or the one used in previous publications [2-5]:

$$
d(x, t)=\cos ^{2}\left(\frac{x-V t-s_{l}}{2 a}\right)
$$

which allows to alleviate doubts related to the solution regularity.

Geometrical irregularities appearing on the rolling surface of the rail head generates additional forces (random in time and space - along the rail). Modelling of this random part $P_{R}(x, t)$ is the main issue investigated in this paper.

In previous papers, a special draw procedure was shown, leading to graphical representation of irregularities described in terms of technical requirements for operational rail networks [8]. However, the representation of forces generated by these irregularities remained an open problem.

Further investigations allowed to improve the developed procedure and to find formula that can be included directly in Wolfram Mathematica codes built for the analysis of the rail track response to moving train. Preliminary tests show that the obtained results can be effectively used in coding. However, this paper presents general assumptions of this procedure only, with some numerical examples. The analysis of solution for the rail track response with inclusion of the developed random part of forces arising from moving train is left for further work and will be presented in future papers.

\section{Random irregularities and the additional force}

It is known that the sources of loads generated by moving trains are associated not only with its weight but also with all sorts of imperfections appearing in vehicle and infrastructure. Most of them are modelled with success by using numerical approaches. However, in the field of analytical modelling, they are still undiscovered in the way that could be satisfactorily 
applied in various parametrical analyses. In the case of rail track, one should pay attention to rail deflections between sleepers. This issue has quite reliable models that can be solved analytically. Another problem is coming from sudden changes of foundation stiffness. These phenomena need usually stochastic approaches and therefore their solution becomes difficult. In this paper, special attention is paid to irregularities appearing on the rail head rolling surface (like e.g. corrugation). One can find some results obtained under assumption that this imperfection is "regular" and has cosine shape. In reality, these damages to the rail are distributed randomly. This fact leads to the necessity of stochastic modelling.

In previous paper, a special procedure for modelling of random rail track imperfections was shown along with discussion of its limitations and some numerical examples [8]. This was the first step to model the additional force appearing during passage of train on corrugated rail track. The draw procedure described in this paper is repeated below as it can be used for obtaining an analytical approximation of the investigated force.

A special code is prepared in Microsoft Excel software with possibility of import of its results to Wolfram Mathematica environment. This is because Wolfram Mathematica allows analytical computations and was already used in modelling of several rail track structures, including simplified models mentioned in section 2. Therefore having specially written formula for direct application to previously developed and validated codes would be highly appreciated.

The draw procedure is based on the following assumptions:

- The length of the beam with imperfections is finite but the length of beam remains infinite.

- $\quad$ The step of draw in $x$ direction is fixed, although it can be made random.

- The amplitude of imperfection is limited (finite), although it can be free of limits (in this case, however, one can obtain random results far from real situations).

- The amplitude between two consecutively selected points changes within some limits (fixed value).

- The amplitude of imperfection and the direction of its change at consecutive points is randomly chosen but in the way that all above conditions are fulfilled.

(a)

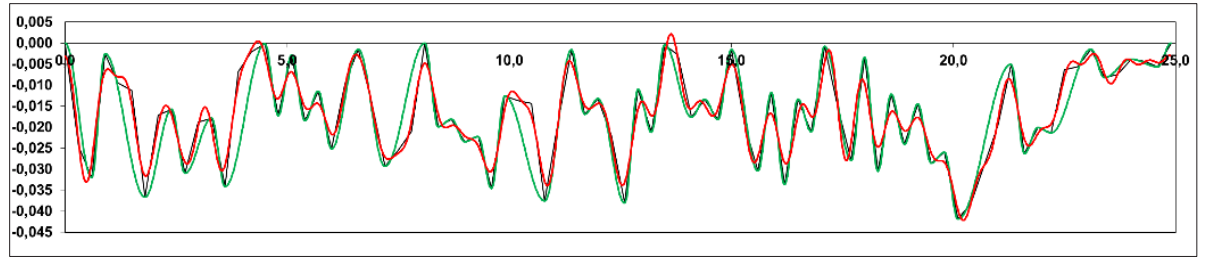

(b)

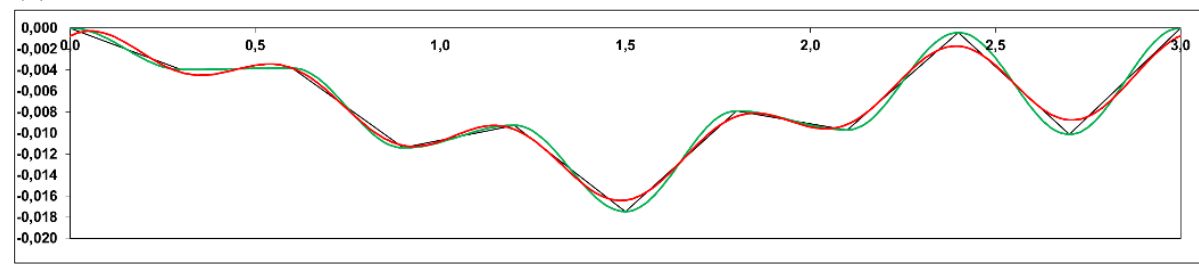

Figure 1. Random data - black line, Fourier approximation - red line, (number of Fourier coefficients: 10), cosine approximation - green line: (a) $25 \mathrm{~m}$; (b) $3 \mathrm{~m}$. 
This procedure is described in detail in [8]. The Fourier series was applied to approximate drawn function, leading to analytical formula applicable in further calculations. Unfortunately, the number of terms in Fourier series makes the procedure ineffective (the system of differential equations must be solved for each of series terms separately).

In this paper, one proposes to replace the Fourier series by cosine functions (Figure 1). This idea reduces extremely the number of needed calculations. Instead of a sum of functions, it gives only one function (a piecewise function), which is, in addition, continuous.

The next step proposed in this paper is double differentiation of the obtained continuous piecewise function with respect to the time variable. This process gives acceleration of additional vibrations generated by irregularities. Because the piecewise function to be differentiated actually does not have a derivative, the derivation must be made in separated open intervals (between drawn points) and another approximation using cosine function should be applied (Figure 2).

(a)

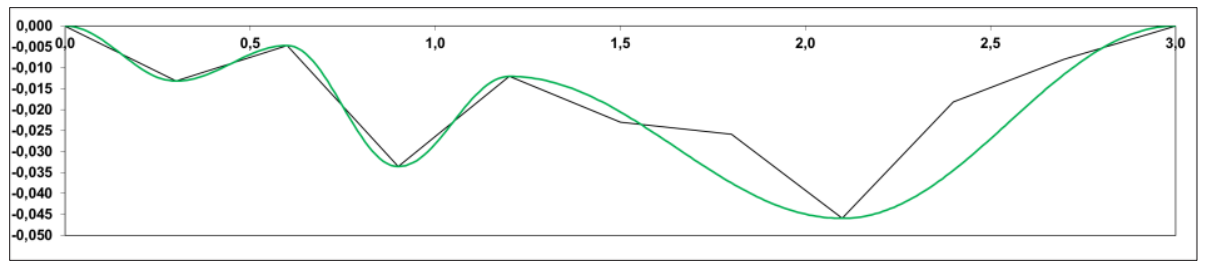

(b)

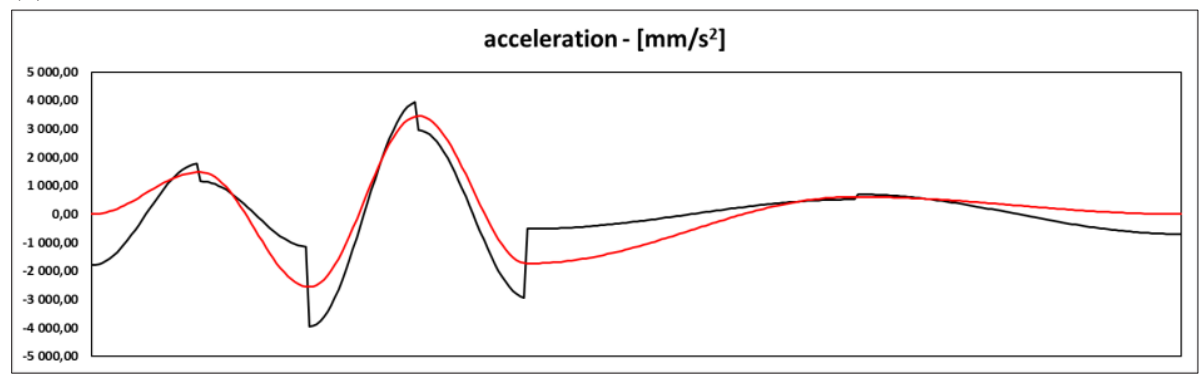

Figure 2. (a) random data - black line, cosine approximation - green line; (b) second derivative of piecewise function - black line, cosine approximation - red line (the length of beam: $3 \mathrm{~m}$ ).

This procedure gives another piecewise function that can be directly used in Wolfram Mathematica code. This function multiplied by some parameter associated with the mass responsible for the force generation can give approximation of the sought random force generated by random irregularities. Nevertheless, estimation of this force still remains an open problem, especially that it is different for each interval defining the piecewise function. Some numerical experiments and comparison of results with measurements might lead to analytical approximation of the mass factor responsible for the investigated random force.

\section{Conclusions}

Special procedure for random irregularities of rail head rolling surface, developed in previous paper, is improved by more effective approximation based on cosine function application. In addition, an approximate solution for the force generated by these irregularities is proposed. The obtained solution can be directly used in analytical beam-foundation models of rail track. 
Estimation of the force value needed for numerical calculation of the rail track response remains an open problem.

\section{References}

[1] W. Czyczula, P. Koziol, D. Kudla and S. Lisowski, J. Vib. Control 23, 18, 2989-3006 (2017)

[2] P. Koziol, Proceedings of ICoEV2015, International Conference on Engineering Vibration, Ljubljana, Slovenia, 7-10 September 2015, paper 273, (2015)

[3] P. Koziol, Proceedings of the Fifteenth International Conference on Civil, Structural and Environmental Engineering Computing, Civil-Comp Press, Stirlingshire, UK, Paper 199 (2015)

[4] P. Koziol, Mech. Syst. Signal Process., 79, 174-181 (2016)

[5] P. Koziol, J. Theor. Appl. Mech., 52, 3, 687-697 (2014)

[6] P. Koziol, Z. Hryniewicz, CC2009, Proceedings of the Twelfth International Conference on Civil, Structural and Environmental Engineering Computing, CivilComp Press, Stirlingshire, UK, paper 42 (2009)

[7] P. Koziol, M.M. Neves, SAV, 19, 1009-1018 (2012)

[8] P. Koziol, D. Kudla, Proceedings of the MPSVA2018 conference, IOP Conference Series (in print) 